Math. Model. Nat. Phenom.

Vol. 6, No. 4, 2011, pp. 77-86

DOI: $10.1051 / \mathrm{mmnp} / 20116404$

\title{
Dense Granular Poiseuille Flow
}

\author{
E. Khain * \\ Department of Physics, Oakland University, Rochester MI 48309, USA
}

\begin{abstract}
We consider a dense granular shear flow in a two-dimensional system. Granular systems (composed of a large number of macroscopic particles) are far from equilibrium due to inelastic collisions between particles: an external driving is needed to maintain the motion of particles. Theoretical description of driven granular media is especially challenging for dense granular flows. This paper focuses on a gravity-driven dense granular Poiseuille flow in a channel. A special focus here is on the intriguing phenomenon of fluid-solid coexistence: a solid plug in the center of the system, surrounded by fluid layers. To find and analyze various flow regimes, a multi-scale approach is taken. On macro scale, granular hydrodynamics is employed. On micro scale, eventdriven molecular dynamics simulations are performed. The entire phase diagram of parameters is explored, in order to determine which flow regime occurs in various regions in the parameter space.
\end{abstract}

Key words: granular matter, poiseuille flow, shear flow, md simulations, two-phase flow AMS subject classification: 76T25, 76F10, 76E99

\section{Introduction}

Granular materials present a fascinating example of intrinsically non-equilibrium systems. Granular matter can be modeled as a system composed of a large number of macroscopic particles that collide inelastically, losing energy in each binary collision. Therefore, an external driving force is needed to pump energy into the system and maintain the motion of particles. In the middle of the 1980-s, a hydrodynamic description of granular flow was developed by Haff [1] and Jenkins and Richman [2]. Working along the lines of Enskog theory [3], they started from the "microscopic"

\footnotetext{
*Corresponding author. E-mail: khain@oakland.edu
} 
dynamics of inelastic hard sphere model and kinetic theory of gases [4] and arrived at a granular hydrodynamics. The physics community became interested in the subject in the early 1990-s after a pioneering work by Goldhirsch and Zanetti [5], who discovered the clustering instability [5] by employing both granular hydrodynamics and molecular dynamics simulations. When I started working on problems in granular dynamics, the work by Goldhirsch and Zanetti [5] was one of the first papers I read. As a matter of fact, my first paper on granular materials was on clustering instability in driven granular matter [6], and the mechanism of this phase-separation instability [7] is somewhat similar to the mechanism explained in the work of Isaac Goldhirsch. I was privileged to meet with Isaac many times; I even commuted from Jerusalem to Tel-Aviv to attend his course on kinetic theory of gases. This paper is dedicated to his memory.

The hydrodynamic equations should be accompanied by proper constitutive relations (expressions for transport coefficients, pressure, and inelastic heat loss). For dilute and moderately dense flows, these relations have been derived from kinetic theory [1,2] (though it is still a subject of ongoing research [8]), while for really dense systems the constitutive relations remain unknown. Therefore, dense granular flows still present a significant challenge for theorists $[9,10]$. During the last decade, several attempts to extend the theory to high densities have been made $[11,12,13,14,15,16]$. A significant progress has been achieved recently, when the coefficient of shear viscosity was found to diverge at a lower density than other constitutive relations [17]: inelastic heat losses, thermal conductivity, and pressure diverge at the crystalline close packing density $n_{\max }$, while shear viscosity becomes infinite at a smaller density $n_{\eta}<n_{\max }$. Recently, modified formulas for constitutive relations (accounting for viscosity divergence) have been proposed and justified in molecular dynamics simulations [18], see also [19].

As was recently shown for dense Couette flows, viscosity divergence at $n_{\eta}<n_{\max }$ may result in coexistence of fluid and solid phases $[18,20,21,22]$. It is well known that the basic granular Couette flow with uniform density and temperature and linear velocity profile becomes unstable when inelasticity of particle collisions exceeds a critical threshold, see, for example, Ref. [23]. This instability leads to density inhomogeneities in the system: some regions become denser, while others become more dilute. If the mean density in the system is sufficiently high, the density in denser regions might become larger than the density of viscosity divergence, $n>n_{\eta}$. This dense region becomes a "plug" (a solid cluster): the velocity can not vary across the cluster. This plug-flow regime had been first observed in Ref. [24], but its theoretical explanation was given only recently $[18,20,21,22]$. Notice that the density and temperature do vary across the solid cluster (since $n<n_{\max }$ ), and the particles are fluidized. Regions with $n<n_{\eta}$ behave like fluid; this explains the fluid-solid coexistence in dense granular Couette flow.

While granular Couette flows have attracted much attention, there are only a few works on granular Poiseuille flow [25, 26, 27, 28, 29, 30, 31], and the problem of fluid-solid coexistence in dense Poiseuille flow remains unresolved. In a classical Poiseuille flow, a thermostat is needed to organize heat flux out of the system, in order to compensate for viscous heating. In granular media, there is no need in thermostat: particles collide inelastically, so that viscous heating is balanced by inelastic heat losses. Therefore, steady flow regimes can be achieved. These steady states are intrinsically far from equilibrium due to the constant heat flux throughout the system. The plugflow regime is particularly intriguing, see Figure 1 . Here, the flow is in the horizontal $x$-direction 


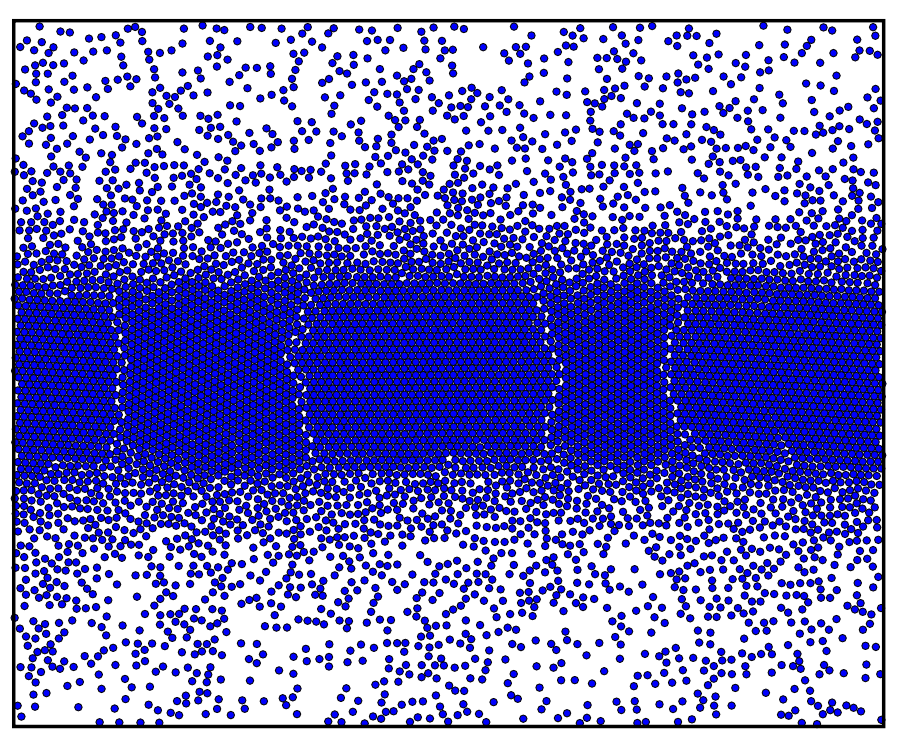

Figure 1: Snapshot of the two-phase flow for $r=0.95, N=6480$, and $f=0.50, L / H=$ 1.25. A large stripe-like cluster in the middle moves as a whole, showing solid-like behavior. The granulate is fluidized everywhere including inside the cluster: the density is smaller than that of close packing, and the granular temperature is larger than zero. However, since $n_{\eta}<n$ inside the plug, the horizontal flow velocity does not vary across the cluster. The hydrodynamics heat loss parameter here is $R=2349.3$, so the parameters locate the system in region $A$ in Fig. 2 far from the transition border.

(the direction of gravity); the horizontal velocity is zero at the walls $y=-H / 2$ and $y=H / 2$. This is a two-phase flow, where a dense "solid-like" cluster is surrounded by two fluid layers, similarly to what was recently found in the Couette geometry [18, 20, 21, 22]. The velocity does not vary across the cluster; the cluster moves as a solid block. Various two-phase regimes were recently observed in molecular dynamics simulations [32, 33]; however, their theoretical description is lacking. In the current project, the granular hydrodynamics with proper constitutive relations was employed for quantitative description of dense Poiseuille flow (a two-dimensional gravity driven flow of granular particles in a channel), including the regime of fluid-solid coexistence. In addition, event-driven molecular dynamics (MD) simulations were performed. In simulations, grains are modeled as inelastic hard spheres, which follow ballistic trajectories between instantaneous and binary collisions. 


\section{Model description and granular hydrodynamics}

Consider a granular flow in a two-dimensional geometry (in a channel), see Fig. 1. The grains are assumed to be inelastic hard disks that interact with each other via a hard-sphere potential. An ensemble of $\mathrm{N}$ inelastically colliding hard disks of unit mass and diameter $\mathrm{d}$ is driven by gravity along the channel (in $\mathrm{x}$ - direction). The "upper" wall is located at $y=\mathrm{H} / 2$, the "lower" wall is located at $y=-H / 2$. The two walls are at rest. Typically, the initial conditions are the following: particles have random velocities and are distributed homogeneously over the system. Boundary conditions in the $\mathrm{x}$-direction are periodic. In the y-direction no slip boundary conditions are implemented. The collisions of particles with the walls are assumed to be elastic (no-heat-flux boundary conditions).

The Navier-Stokes granular hydrodynamics operates with coarse grained quantities: number density of grains, $n(\mathbf{r}, t)$, granular temperature $T(\mathbf{r}, t)$, and mean flow velocity $v(\mathbf{r}, t)[1,2]$ :

$$
\begin{aligned}
& \frac{d n}{d t}+n \nabla \cdot \mathbf{v}=0, \\
& n \frac{d \mathbf{v}}{d t}=\nabla \cdot \mathbf{P}+n g \hat{\mathbf{x}}, \\
& n \frac{d T}{d t}=-\nabla \cdot \mathbf{Q}+\mathbf{P}: \nabla \mathbf{v}-\Gamma
\end{aligned}
$$

Here $\mathbf{P}$ is the stress tensor, $\mathbf{Q}$ is the heat flux, $\Gamma$ is inelastic heat losses, and $\hat{\mathbf{x}}$ is a unit vector in the $x$ - direction (the direction of gravity). The stress tensor $\mathbf{P}$ is given by

$$
\mathbf{P}=[-p(n, T)+\mu(n, T) \operatorname{tr}(\mathbf{D})] \mathbf{I}+2 \eta(n, T) \hat{\mathbf{D}},
$$

where $\mathbf{D}=(1 / 2)\left[\nabla v+(\nabla v)^{T}\right]$ is the rate of deformation tensor, $\hat{\mathbf{D}}=\mathbf{D}-\frac{1}{2} \operatorname{tr}(\mathbf{D}) \mathbf{I}$ is the deviatoric part of $\mathbf{D}$, and $\mathbf{I}$ is the identity tensor, $\eta(n, T)$ and $\mu(n, T)$ are the shear and bulk viscosities. The heat flux $\mathbf{Q}$ is given by $\mathbf{Q}=-\kappa(n, T) \nabla T$, where $\kappa(n, T)$ is the coefficient of thermal conductivity. In the limit of nearly elastic collisions, $1-r^{2} \ll 1$, an additional term in the expression for the heat flux, which is proportional to the density gradient $[34,35]$, can be neglected. We also neglect inelastic corrections to transport coefficients $[34,35]$ and replace $1-r^{2}$ factor in the inelastic heat losses term by $2(1-r)$.

Let us now describe the possible steady states $(\partial / \partial t=0)$ of hydrodynamic equations, assuming flow in $x$-direction, zero vertical velocity $(v=0)$ and $\partial / \partial x=0$. The coordinate $y$ will be measured in units of the system "height" $H$ (the distance between the two walls in $y$-direction), the horizontal velocity $u$ will be measured in units of $\sqrt{g H}$, the temperature $T$ in units of $g H$, the density $n$ in units of $n_{\text {max }}$, and the pressure $P$ in units of $n_{\max } g H$. The steady state equations can be written now in the following dimensionless form

$$
\begin{aligned}
& \epsilon \frac{d}{d y}\left(\eta(n, T) \frac{d u}{d y}\right)+n=0, \\
& \frac{d}{d y}\left(\kappa(n, T) \frac{d T}{d y}\right)+\eta(n, T)\left(\frac{d u}{d y}\right)^{2}-R \Gamma(n, T)=0, \\
& p(n, T)=\text { const. }
\end{aligned}
$$


Here $R=(16 / \sqrt{3 \pi})(1-r)(H / d)^{2}$ is the hydrodynamic heat loss parameter, $\epsilon=(\sqrt{3} / 2) d / H$ is the small (Knudsen-like) parameter, and the constitutive relations for thermal conductivity $\kappa(n, T)$, pressure $p(n, T)$, shear viscosity $\eta(n, T)$, and inelastic heat losses $\Gamma(n, T)$ are given using interpolation functions between the dilute and dense limits; the reader is referred to Refs. [18, 22, 19] for more details. It was recently found that while inelastic heat losses, thermal conductivity, and pressure diverge at the close packing density $n_{\max }$, the shear viscosity diverges at a lower density [17]. The authors [17] measured the density of viscosity divergence $n_{\eta}$ (and the corresponding area fraction $\nu_{\eta}$, where $\nu=n \pi d^{2} / 4$ ) in the molecular dynamics simulation of a non-sheared homogeneous freely evolving system of elastic hard disks using Einstein-Helfand expressions [17]. Their result was $\nu_{\eta}=0.71 \pm 0.01$. Then this value $\left(\nu_{\eta}=0.71\right)$ was confirmed in independent molecular dynamics simulations of uniform shear flow [18]; the uniform shear flow can not exist if the area fraction is larger than 0.71 [18]. In the present calculations, I incorporate the viscosity divergence and use the same value of $\nu_{\eta}$. Due to viscosity divergence, the system of equations, describing the solid phase, is somewhat different: since the density inside the cluster is larger than $n_{\eta}$, one needs to demand a constant velocity inside the cluster $[18,22]$. In addition, there is no viscous heating term in the energy balance equation. The resulting system is (compare to Eqs. 2.2):

$$
\begin{aligned}
& u=\text { const } \\
& \frac{d}{d y}\left(\kappa(n, T) \frac{d T}{d y}\right)-R \Gamma(n, T)=0, \\
& p(n, T)=\text { const. }
\end{aligned}
$$

The total number of particles is conserved $\int_{-1 / 2}^{1 / 2} n(y) d y=N /\left(L H n_{\max }\right) \equiv f$ and no-heat-flux and no-slip boundary conditions are assumed: $d T / d y(y=-1 / 2)=d T / d y(y=1 / 2)=u(y=$ $-1 / 2)=u(y=1 / 2)=0$. One needs to match the solutions of Eqs. (2.2) in fluid layers to the solution of Eqs. (2.3) inside the solid cluster. The fluid-solid interfaces are determined from the condition $n=n_{\eta}$, where $n_{\eta}$ is the density of viscosity divergence. At each of these interfaces one needs to demand the continuity of the velocity and temperature profiles, and the continuity of the heat flux (the temperature gradient).

\section{Phase diagram}

Solving Equations (2.2) and (2.3), one can compute the steady flow regimes for different sets of parameters $(R, f, \epsilon)$. Figure 2 shows the $(R, f)$ phase plane with two qualitatively different regions. In region B (below the solid curve), there is a one-phase fluid flow regime, while in region A (above the solid curve), there is fluid-solid coexistence. In the two-phase flow, a solid cluster is surrounded by two fluid layers, as can be seen in Fig. 1. The border $R_{c}(f)$ between regions A and $\mathrm{B}$ is determined by the condition that the maximal density in the system is equal to the density of viscosity divergence, $n_{\eta}$. This border was computed by assuming one-phase flow and solving Eqs. (2.2); note that $R_{c}(f)$ curve does not depend on $\epsilon$. 


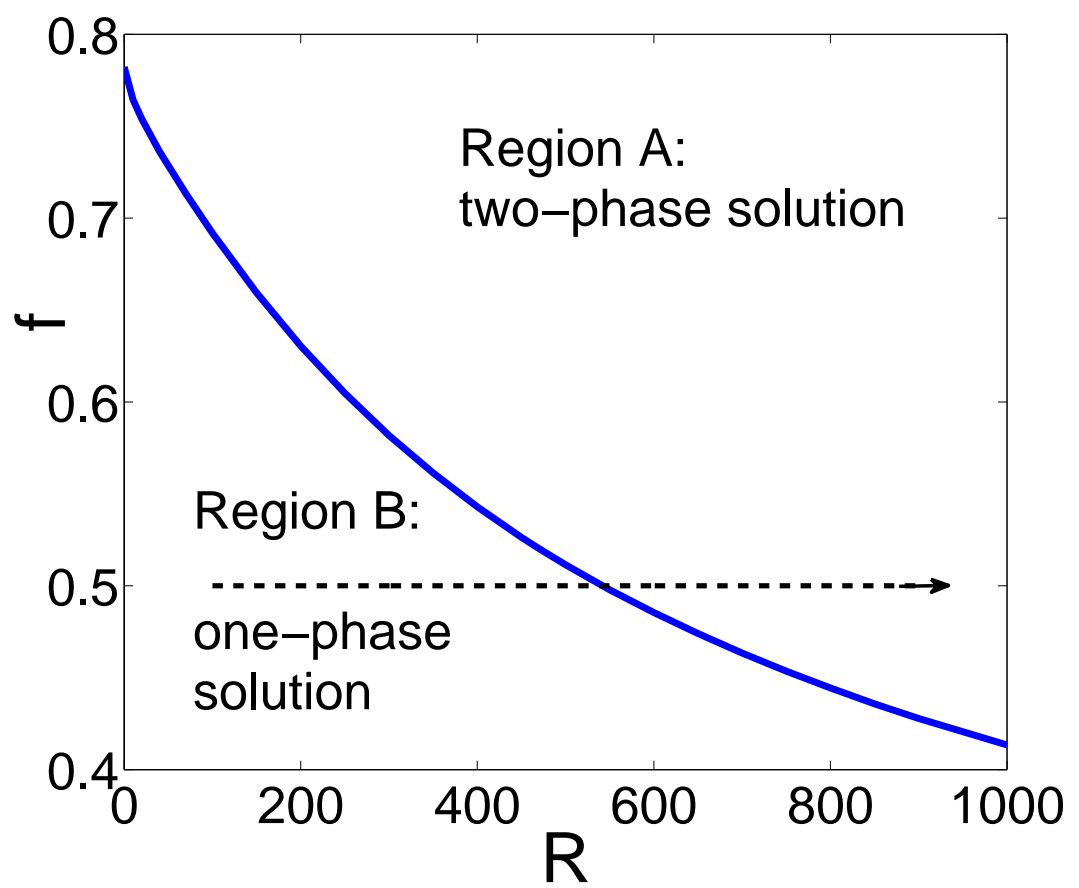

Figure 2: Phase diagram as obtained from numerical solution of steady state hydrodynamic equations. The solid curve computed from Eqs. (2.2) is a border between region of fluid-solid coexistence with the moving plug in the middle (Region A) and a one-phase fluid region (Region $\mathrm{B}$ ). The width of the solid plug, computed along the dotted line $(f=0.5)$, is shown in Fig. 3.

Let us now vary $R$ for the fixed $f$ and $\epsilon$ (as shown in Fig. 2 by the dashed line) and measure the width of the solid plug, $w$, in the middle of the system. One would expect that $w=0$ in region $B$ and $w>0$ in region $A$. However, the situation is more complicated close to the transition border between the two regions, as illustrated in Fig. 3, showing the cluster width as a function of $R$. The inset shows that close to the transition, there are three steady state solutions for the same value of $R$. In the first solution, the density everywhere is smaller than $n_{\eta}$, no cluster is formed, so $w=0$. Two other flow regimes are two-phase solutions with $w>0$. The bifurcation diagram suggests that the two-phase flow with smaller $w$ is unstable (dashed line) and the two-phase flow with larger $w$ is stable (solid line). A similar bistability was observed in Ref. [22] for Couette granular flow, both theoretically and in MD simulations. The solution of Equations (2.2) and (2.3) shows that the bistability occurs for $535.2<R<541.8$. When $R>541.8$, there is only one steady flow solution: the two-phase regime; one can see that the width of the cluster increases with $R$. Two squares show the results of MD simulations, in a reasonable agreement with the theoretical results. When the width of the channel (along the stream-wise direction) becomes larger, various two-dimensional flow patterns (with explicit dependence on $x$ - and $y$ - coordinates) can appear $[32,33]$. One such flow regime is an S-shaped density wave along the channel, see Fig. 4. This snapshot corresponds 


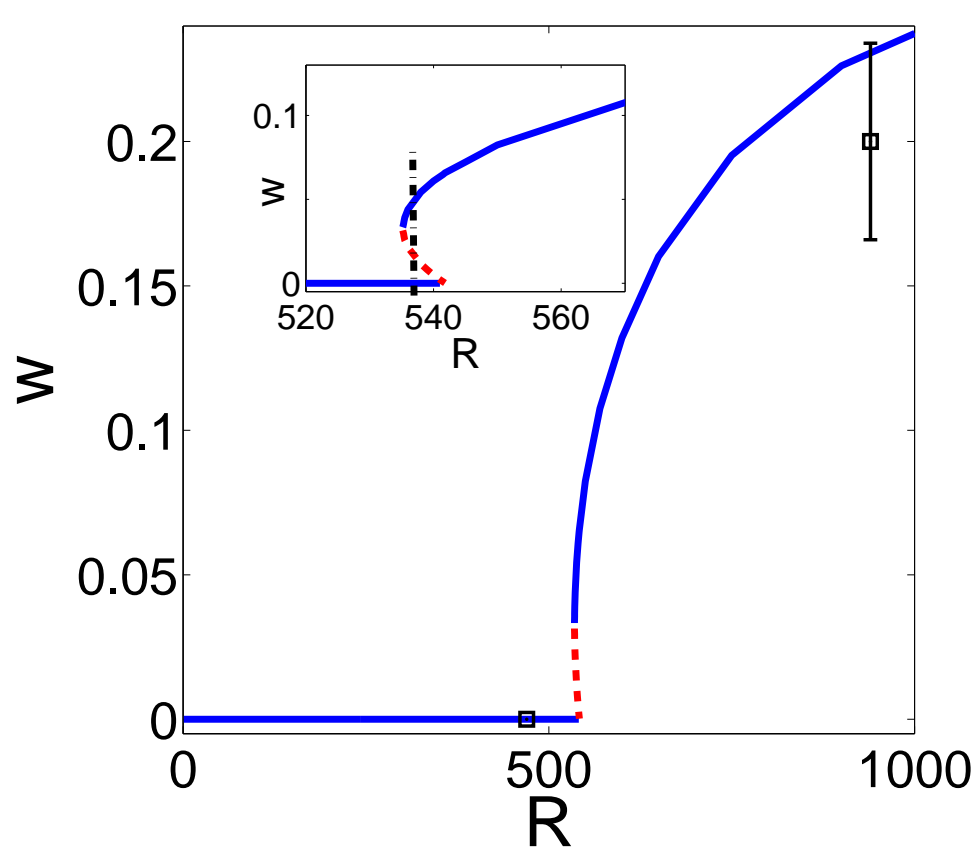

Figure 3: The width of the solid plug, computed along the dotted line $(f=0.5)$ in Fig. 2. The inset shows a magnified picture of the transition region. One can see that in some interval of $R$, three flow regimes are possible for the same value of $R$, see text.

to the same parameters as in Fig. 1, except for the system width $L$ and the total number of particles $N$, which were increased by factor 3 . This is also a two-phase flow: the density inside the S-shaped cluster is higher than the density of viscosity divergence. A possible direction of future research is a linear stability analysis of an $x$ - independent dense two-phase flow. This analysis can show when the $x$ - independent two-phase solution may become unstable and give rise to the two-phase density wave. However, the analysis is technically challenging, since the interface between the fluid-like and solid-like regions becomes curved.

\section{Summary}

This study focuses on a dense fluidized granular flow in Poiseuille geometry in the framework of the simplest model of fluidized granular media: inelastic hard sphere model in two dimensions. An intriguing plug-flow regime is investigated both theoretically by using Navier-Stokes granular hydrodynamics, and in event-driven molecular dynamics simulations. The plug formation can be explained by the nontrivial dependence of the coefficient of shear viscosity on density: the shear viscosity diverges at the density smaller than the density of crystalline close packing $n_{\eta}<$ 


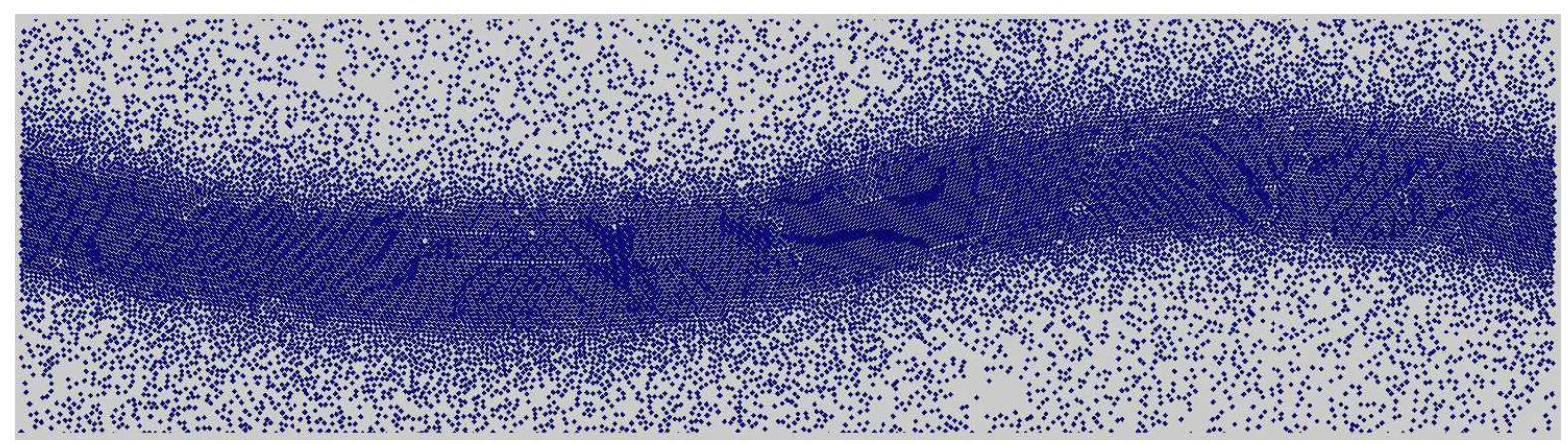

Figure 4: Snapshot of the wavy two-phase flow for the same average density $f=0.50$ and restitution coefficient $r=0.95$ as in Fig. 1. The wavy patterns appear when the aspect ratio $L / H$ becomes larger than some threshold value. $N=19440$ and $L / H=3.75$.

$n_{\max }$. This explains the observed solid-fluid coexistence: the density in the solid-like plug is above $n_{\eta}$, while in the fluid-like regions it is below $n_{\eta}$. Importantly, the particles inside the cluster are fluidized, and the granular temperature is larger than zero. Similar fluid-solid coexistence was observed in dense granular Couette flows [20, 18, 22].

Particles inside the plug form an ordered crystalline structure, though the density is smaller than $n_{\max }$. Does this happen because the density of viscosity divergence $n_{\eta}$ is very close to the density of the melting point? Or may be ordering is a feature of inelastically colliding hard disks, since it is also observed in systems without any shear? Further studies are needed to address these questions.

\section{References}

[1] P. K. Haff. Grain flow as a fluid-mechanical phenomenon. J. Fluid Mech., 134 (1983), 401430.

[2] J. T. Jenkins, M. W. Richman. Kinetic theory for plane flows of a dense gas of identical, rough, inelastic, circular disks. Phys. Fluids, 28 (1985), 3485-94.

[3] S. Chapman, T. G. Cowling. The Mathematical Theory of Non-Uniform Gases. Cambridge Univ. Press, Cambridge, 1990.

[4] N. V. Brilliantov, T. Pöschel. Kinetic Theory of Granular Gases. Oxford University Press, Oxford, 2004; Granular Gas Dynamics edited by T. Pöschel, N. Brilliantov. Springer, Berlin, 2003.

[5] I. Goldhirsch, G. Zanetti. Clustering instability in dissipative gases. Phys. Rev. Lett., 70 (1993), 1619-1622. 
[6] E. Khain, B. Meerson. Symmetry-breaking instability in a prototypical driven granular gas. Phys. Rev. E, 66 (2002), 021306.

[7] E. Khain, B. Meerson, P. V. Sasorov. Phase diagram of van der Waals-like phase separation in a driven granular gas. Phys. Rev. E, 70 (2004), 051310.

[8] A. Baskaran, J. W. Dufty, J. J. Brey. Transport coefficients for the hard-sphere granular fluid. Phys. Rev. E, 77 (2008), 031311.

[9] I. S. Aranson, L. S. Tsimring. Patterns and collective behavior in granular media: Theoretical concepts. Rev. Mod. Phys., 78 (2006), 641-692.

[10] I. Goldhirsch. Rapid granular flows. Annu. Rev. Fluid Mech., 35 (2003), 267-293.

[11] E. L. Grossman, T. Zhou, E. Ben-Naim. Towards granular hydrodynamics in two dimensions. Phys. Rev. E, 55 (1997), 4200-4206.

[12] S. Luding. Global equation of state of two-dimensional hard sphere systems. Phys. Rev. E, 63 (2001), 042201.

[13] B. Meerson, T. Pöschel, Y. Bromberg. Close-packed floating clusters: Granular hydrodynamics beyond the freezing point? Phys. Rev. Lett., 91 (2003), 024301.

[14] P. Eshuis, K. van der Weele, D. van der Meer, D. Lohse. Granular Leidenfrost effect: Experiment and theory of floating particle clusters. Phys. Rev. Lett., 95 (2005), 258001.

[15] L. Bocquet, W. Losert, D. Schalk, T. C. Lubensky, J. P. Gollub. Granular shear flow dynamics and forces: Experiment and continuum theory. Phys. Rev. E, 65 (2002), 011307.

[16] L. Bocquet, J. Errami, T. C. Lubensky. Hydrodynamic model for a dynamical jammed-toflowing transition in gravity driven granular media. Phys. Rev. Lett., 89 (2002), 184301.

[17] R. Garcia-Rojo, S. Luding, J. J. Brey. Transport coefficients for dense hard-disk systems. Phys. Rev. E, 74 (2006), 061305.

[18] E. Khain. Hydrodynamics of fluid-solid coexistence in dense shear granular flow. Phys. Rev. E, 75 (2007), 051310.

[19] S. Luding. Towards dense, realistic granular media in 2D. Nonlinearity, 22 (2009), No. 12, R101-R146.

[20] E. Khain, B. Meerson. Shear-induced crystallization of a dense rapid granular flow: Hydrodynamics beyond the melting point. Phys. Rev. E, 73 (2006), 061301.

[21] M. Alam, P. Shukla, S. Luding. Universality of shear-banding instability and crystallization in sheared granular fluid. J. Fluid Mech., 615 (2008), 293. 
[22] E. Khain. Bistability and hysteresis in dense shear granular flow. Europhys. Lett., 87 (2009), 14001.

[23] M. Alam, P. R. Nott. Stability of plane Couette flow of a granular material. J. Fluid Mech., 377 (1998), 99-136.

[24] M. Alam, S. Luding. First normal stress difference and crystallization in a dense sheared granular fluid. Phys. Fluids, 15 (2003), 2298-2312.

[25] C. H. Wang, R. Jackson, S. Sundaresan. Instabilities of fully developed rapid flow of a granular material in a channel. J. Fluid Mech., 342 (1997), 179-197.

[26] C. H. Wang, Z. Q. Tong. On the density waves developed in gravity channel flows of granular materials. J. Fluid Mech., 435 (2001), 217-246.

[27] C. Denniston, H. Li. Dynamics and stress in gravity-driven granular flow. Phys. Rev. E, 59 (1999), 3289-3292.

[28] J. J. Drozd, C. Denniston. Simulations of collision times in gravity-driven granular flow. Europhys. Lett., 76 (2006), 360.

[29] J. C. Tsai, W. Losert, G. A. Voth, J. P. Gollub. Two-dimensional granular Poiseuille flow on an incline: Multiple dynamical regimes. Phys. Rev. E, 65 (2002), 011306.

[30] K. C. Vijayakumar, M. Alam. Velocity distribution and the effect of wall roughness in granular Poiseuille flow. Phys. Rev. E, 75 (2007), 051306.

[31] V. Chikkadi, M. Alam. Slip velocity and stresses in granular Poiseuille flow via event-driven simulation. Phys. Rev. E, 80 (2009), 021303.

[32] E. D. Liss, S. L. Conway, B. J. Glasser. Density waves in gravity-driven granular flow through a channel. Phys. Fluids, 14 (2002), 3309-3326.

[33] M. Alam, V. Chikkadi, V. K. Gupta. Density waves and the effect of wall roughness in granular Poiseuille flow: Simulation and linear stability. EPJ St, 179 (2009), 69-90.

[34] J. J. Brey, F. Moreno, J. W. Dufty. Model kinetic equation for low-density granular flow. Phys. Rev. E, 54 (1996), 445-456.

[35] N. Sela, I. Goldhirsch. Hydrodynamic equations for rapid flows of smooth inelastic spheres, to Burnett order. J. Fluid Mech., 361 (1998) 41-74. 